\title{
A Speed-Up and Speed-Down Strategy for Swarm Optimization
}

\author{
Haopeng Zhang \\ Department of Mechanical \\ Engineering \\ Texas Tech University \\ Lubbock, TX79409-1021 \\ haopeng.zhang@ttu.edu
}

\author{
Fumin Zhang \\ School of Electrical and \\ Computer Engineering \\ Georgia Institute of \\ Technology \\ Atlanta, GA 30332-0250 \\ fumin@gatech.edu
}

\author{
Qing Hui \\ Department of Mechanical \\ Engineering \\ Texas Tech University \\ Lubbock, TX 79409-1021 \\ qing.hui@ttu.edu
}

\begin{abstract}
In this paper, inspired by speed-up and speed-down (SUSD) mechanism observed by the fish swarm avoiding light, an SUSD strategy is proposed to develop new swarm intelligence based optimization algorithms to enhance the accuracy and efficiency of swarm optimization algorithms. By comparing with the global best solution, each particle adaptively speeds up and speeds down towards the best solution. Specifically, a new directed speed term is added to the original particle swarm optimization (PSO) algorithm or other PSO variations. Due to the SUSD mechanism, the algorithm shows a great improvement of the accuracy and convergence rate compared with the original PSO and other PSO variations. The numerical evaluation is provided by solving recent benchmark functions in IEEE CEC 2013.
\end{abstract}

\section{Categories and Subject Descriptors}

G.1.6 [Optimization]: Unconstrained optimization

\section{Keywords}

Swarm intelligence

\section{INTRODUCTION}

Particle swarm optimization (PSO), firstly proposed in [1], has been widely used in various disciplines. As a swarm intelligence based optimization algorithm, a particle in PSO updates its velocity by comparing the difference between current position and local best position found by itself and the difference between current position and the global best position among all the particles, and then moves into the next position, after which the local best position and the global position will be updated simultaneously.

Permission to make digital or hard copies of part or all of this work for personal or classroom use is granted without fee provided that copies are not made or distributed for profit or commercial advantage, and that copies bear this notice and the full citation on the first page. Copyrights for third-party components of this work must be honored. For all other uses, contact the owner/author(s). Copyright is held by the author/owner(s).

GECCO'14, July 12-16, 2014, Vancouver, BC, Canada.

ACM 978-1-4503-1964-5/14/07.

http://dx.doi.org/10.1145/2598394.2602285.
The update formulas for the PSO algorithm are shown in the following equation.

$$
\begin{aligned}
\mathbf{v}_{k+1}^{i, j} & =w \mathbf{v}_{k}^{i, j}+b_{1} r_{1}\left(\mathbf{g}_{l o c, i}^{j}-\mathbf{x}_{k}^{i, j}\right)+b_{2} r_{2}\left(\mathbf{g}_{k}^{j}-\mathbf{x}_{k}^{i, j}\right) \\
\mathbf{x}_{k+1}^{i, j} & =\mathbf{x}_{k}^{i, j}+\mathbf{v}_{k+1}^{i, j}, \quad i=1, \ldots, q, \quad j=1, \ldots, n
\end{aligned}
$$

where $\mathbf{x}_{k}^{i, j}$ and $\mathbf{v}_{k}^{i, j}$ are the $j$ th position and velocity element of particle $i$ at iteration $k$, respectively, $\mathbf{g}_{k}^{j}$ is the $j$ th element of the global best solution among all the particles and $\mathbf{g}_{l o c, i}^{j}$ is the $j$ th element of local best solution found by particle $i$, and $w, b_{1}, b_{2}, r_{1}$ and $r_{2}$ are positive constants.

Due to the great success of PSO, lots of research efforts have been conducted to improve the performance in both accuracy and efficiency. In this research, observed from the fish swarm behavior [3], a speed-up and speed-down (SUSD) mechanism is proposed for PSO as an extra force for the velocity towards both local and global best solutions. Moreover, this SUSD mechanism can be easily integrated into other PSO variants for performance enhancement. As an example, multiagent coordination optimization (MCO) [4] is introduced and illustrated by using the SUSD mechanism.

This work was supported by the Defense Threat Reduction Agency under Grants HDTRA1-10-1-0090 and HDTRA 1-13-1-0048.

\section{SPEED-UP AND SPEED-DOWN MECH- ANISM}

Based on the observation that a fish in a group speeds up when the light intensity at its current position is relatively high and slows down as the light intensity decreases [3], a SUSD mechanism is proposed as follows.

$$
S U S D=\lambda_{1}\left(f_{j}\left(\mathbf{g}_{k}^{j}\right)-f_{j}\left(\mathbf{x}_{k}^{i, j}\right)\right) \operatorname{sign}\left(\mathbf{g}_{k}^{j}-\mathbf{x}_{k}^{i, j}\right)
$$

where $f_{j}(z)=\left.f\left(x_{1}, \ldots, x_{n}\right)\right|_{x_{j}=z}, i=1, \ldots, q, j=1, \ldots, n$, $q$ is the number of particles, $n$ is the dimension of the objective function $f: \mathbb{R}^{n} \rightarrow \mathbb{R}, \lambda_{1}<0$ is a constant, $\operatorname{sign}(x)=1$ if $x>0, \operatorname{sign}(x)=-1$ if $x<0$, and $\operatorname{sign}(0)=0$.

\subsection{SUSD for the PSO algorithm}

Now this SUSD mechanism is applied to the PSO algorithm, and the update formulas for position and velocity are 
proposed in Equations (2-3).

$$
\begin{aligned}
\mathbf{v}_{k+1}^{i, j}= & \mathbf{v}_{k}^{i, j}+b_{1} r_{1}\left(\mathbf{g}_{l o c, i}^{j}-\mathbf{x}_{k}^{i, j}\right)+b_{2} r_{2}\left(\mathbf{g}_{k}^{j}-\mathbf{x}_{k}^{i, j}\right) \\
& +\lambda_{1}\left(f_{j}\left(\mathbf{g}_{k}^{j}\right)-f_{j}\left(\mathbf{x}_{k}^{i, j}\right)\right) \operatorname{sign}\left(\mathbf{g}_{k}^{j}-\mathbf{x}_{k}^{i, j}\right) \\
\mathbf{x}_{k+1}^{i, j}= & \mathbf{x}_{k}^{i, j}+\mathbf{v}_{k+1}^{i, j}
\end{aligned}
$$

\subsection{SUSD for the MCO algorithm}

Multiagent coordination optimization (MCO) [4] is a novel swarm optimization algorithm by introducing the velocity consensus protocol and communication topology between particles into a PSO-like algorithm. The update formulas for position and velocity in MCO are shown in Equations $(4-6)$.

$$
\begin{aligned}
\mathbf{v}_{k+1}^{i, l}= & w \mathbf{v}_{k}^{i, j}+\check{\mu} \sum_{j \in \mathcal{N}_{i}}\left(\mathbf{x}_{k}^{j, l}-\mathbf{x}_{k}^{i, l}\right)+\check{\eta} \sum_{j \in \mathcal{N}_{i}}+\left(\mathbf{v}_{k}^{j, l}-\mathbf{v}_{k}^{i, l}\right) \\
& \check{\kappa}\left(\mathbf{g}_{k}^{l}-\mathbf{x}_{k}^{i, l}\right) \\
\mathbf{x}_{k+1}^{i}= & \mathbf{x}_{k}^{i}+\mathbf{v}_{k+1}^{i} \\
\mathbf{g}_{k+1}^{l}= & \mathbf{g}_{k}^{l}+\check{\kappa}\left(\mathbf{g}_{l o c, i}^{l}-\mathbf{g}_{k}^{l}\right)
\end{aligned}
$$

where $i=1, \ldots, q, l=1, \ldots, n, \check{\eta} \sim U(0,2 \eta), \check{\mu} \sim(0,2 \mu)$, $\check{\kappa} \sim(0,2 \kappa), \eta, \mu, \kappa>0$ are constants, $U(\cdot, \cdot)$ is the uniform distribution, $\mathcal{N}_{i}=\{j \in \mathcal{V}:\{i, j\} \in \mathcal{E}\}$ is the set of neighbors of node $i$ and $\mathcal{E} \subseteq \mathcal{V} \times \mathcal{V}$ denotes the set of edges, which is the communication links between two particles, and the set of nodes $\mathcal{V}=\{1, \ldots, q\}$ denotes the index of the particles. Inspired by the SUSD mechanism, the SUSD-MCO algorithm is proposed in Equations (7-9).

$$
\begin{aligned}
\mathbf{v}_{k+1}^{i, l}= & w \mathbf{v}_{k}^{i, j}+\check{\mu} \sum_{j \in \mathcal{N}_{i}}\left(\mathbf{v}_{k}^{j, l}-\mathbf{v}_{k}^{i, l}\right) \\
& +\check{\mu} \sum_{j \in \mathcal{N}_{i}}\left(\mathbf{x}_{k}^{j, l}-\mathbf{x}_{k}^{i, l}\right)+\check{\kappa}\left(\mathbf{g}_{k}^{l}-\mathbf{x}_{k}^{i, l}\right) \\
& +\lambda_{1}\left(f_{l}\left(\mathbf{g}_{k}^{l}\right)-f_{l}\left(\mathbf{x}_{k}^{i, l}\right)\right) \operatorname{sign}\left(\mathbf{g}_{k}^{l}-\mathbf{x}_{k}^{i, l}\right) \\
\mathbf{x}_{k+1}^{i}= & \mathbf{x}_{k}^{i}+\mathbf{v}_{k+1}^{i} \\
\mathbf{g}_{k+1}^{l}= & \mathbf{g}_{k}^{l}+\check{\kappa}\left(\mathbf{g}_{l o c, i}^{l}-\mathbf{g}_{k}^{l}\right)
\end{aligned}
$$

where $l=1, \cdots, n$ and $i=1, \ldots, q$.

\section{NUMERICAL EVALUATION}

For numerical evaluation, 1000 particles are used to solve the 30-dimension benchmark functions in [2]. Specifically, we use the shifted sphere function, rotated Rosenbrock function, and rotated Griewank function to test the proposed SUSD mechanism. The shifted optimal solution is shown as $X^{*}$, where $X^{*}=\left[\begin{array}{lllll}98.7900 & 17.0400 & 25.7800 & 39.6800 & 7.4000\end{array}\right.$ $\begin{array}{lllllll}68.4100 & 40.2400 & 98.2800 & 40.2200 & 62.0700 & 15.4400 & 38.1300\end{array}$ $\begin{array}{llllllll}16.1100 & 75.8100 & 87.1100 & 35.0800 & 68.5500 & 29.4100 & 53.0600\end{array}$ $\begin{array}{llllllll}83.2400 & 59.7500 & 33.5300 & 29.9200 & 45.2600 & 42.2600 & 35.9600\end{array}$

\begin{tabular}{|c|c|c|}
\hline Function & Min & Max \\
\hline & SUSD-PSO & SUSD-PSO \\
\hline Sphere & $5.9263 \mathrm{E}-6$ & $7.49 \mathrm{E}-2$ \\
\hline Rosenbrock & $2.10 \mathrm{E}-2 \quad 4.5727 \mathrm{E}-7$ & $8.429 \mathrm{E}-1$ \\
\hline Griewank & $2.0379 \mathrm{E} 2 \quad 2.306 \mathrm{E}-1$ & $2.3292 \mathrm{E} 3$ \\
\hline Function & Median & Average \\
\hline & SUSD-PSO & SUSD-PSO \\
\hline Sphere & $2.135 \mathrm{E}-1 \quad 2.1 \mathrm{E}-3$ & $4.543 \mathrm{E}-1 \quad 1.07 \mathrm{E}-2$ \\
\hline Rosenbrock & $1.0811 \mathrm{E}-1$ & $4.1827 \mathrm{E}-4$ \\
\hline Griewank & $\begin{array}{ll}8.5715 \mathrm{E} 2 & 1.2871 \\
\end{array}$ & $1.0002 \mathrm{E} 3$ \\
\hline
\end{tabular}
$55.830074 .250042 .430042 .9400]$. 20 executions of both PSO and SUSD-PSO algorithms solving the three benchmark functions have been conducted, and the results are

\begin{tabular}{|c|c|c|}
\hline Function & Min & Max \\
\hline & SUSD-MCO & SUSD-MCO \\
\hline Sphere & $\begin{array}{ll}2.739 \mathrm{E}-1 & 8.6 \mathrm{E}-3 \\
\end{array}$ & $7.845 \mathrm{E}-1$ \\
\hline Rosenbrock & $\begin{array}{ll}6.5 \mathrm{E}-3 & 4.0371 \mathrm{E}-4 \\
\end{array}$ & $7.32 \mathrm{E}-2$ \\
\hline Griewank & $9.2577 \mathrm{E} 2$ & $1.4405 \mathrm{E} 4 \quad 1.0827 \mathrm{E} 4$ \\
\hline Function & Median & Average \\
\hline & SUSD-MCO & MCO SUSD-MCO \\
\hline Sphere & $6.334 \mathrm{E}-1$ & $5.857 \mathrm{E}-1$ \\
\hline Rosenbrock & $1.62 \mathrm{E}-2$ & $2.09 \mathrm{E}-2$ \\
\hline Griewank & $1.0690 \mathrm{E} 4$ & $1.1243 \mathrm{E} 4$ \\
\hline
\end{tabular}
shown in Table 1. It follows from the simulation results that the SUSD mechanism can largely improve the accuracy of PSO. Moveover, based on the results in Table 2 , the SUSD-MCO algorithm also improves the accuracy of MCO. Due to the page limitation, only the searching trajectories of PSO and SUSD-PSO algorithms solving the shifted sphere function is provided in Figure 1.
Table 1: Comparison between PSO and SUSD-PSO

Table 2: Comparison between MCO and SUSDMCO

\section{REFERENCES}

[1] J. Kennedy and R. Eberhart. Particle swarm optimization. In Proc. IEEE Int. Conf. Neural Networks, pages 1942-1946, 1995.

[2] J. Liang, B. Qu, P. Suganthan, and A. G. Hernández-Díaz. Problem definitions and evaluation criteria for the CEC 2013 special session on real-parameter optimization. Comput. Intell. Lab., Zhengzhou Univ., China, and Nanyang Tech. Univ., Singapore, Technical Report, 2012-12, 2013.

[3] W. Wu, I. D. Couzin, and F. Zhang. Bio-inspired source seeking with no explicit gradient estimation. In Proc. IFAC Workshop on Distributed Estimation and Control in Networked Systems, pages 240-245, 2012.

[4] H. Zhang and Q. Hui. Multiagent coordination optimization: A control-theoretic perspective of swarm intelligence algorithms. In 2013 IEEE Congr. Evolut. Comput., pages 3339-3346, Cancun, Mexico, 2013.

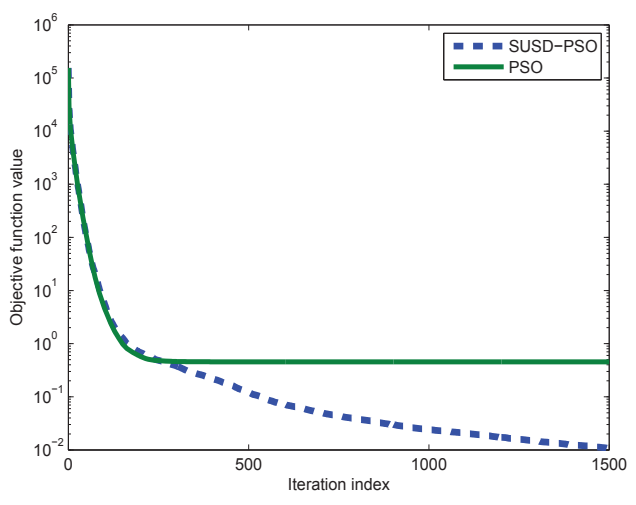

Figure 1: Test function: Shifted sphere function 